\title{
Final results from EROS towards the Galactic Plane
}

\author{
Moniez Marc** \\ Laboratoire de l'Accélérateur Linéaire, IN2P3-CNRS, Université de Paris-Sud, B.P. 34, 91898 \\ Orsay Cedex, France \\ E-mail: moniez@lal.in2p3.fr
}

12.9 million stars have been monitored by EROS during 7 seasons towards 4 directions in the Galactic plane, away from the Galactic center. 27 microlensing event candidates have been found. Estimates of the optical depths from the 22 best events are provided. A first order analysis shows that our observations favour galactic models without a thick disk or a spiral structure. We find that the average microlensing optical depth towards the complete EROS-catalogued stars of the spiral arms is $\tau=0.50_{-.11}^{+.11}$, a number that is stable when moderately varying the selection criteria. As EROS catalog is almost complete (unbiased) until $I_{C}=18.5$, the optical depth estimated for the sub-sample of bright stars with $I_{C}<18.5\left(\tau=0.39_{-.10}^{+.10}\right)$ is easier to interpret.

A more precise interpretation requires either a better knowledge of the distance distribution of the target stars, or a simulation based on a Galactic model. In this purpose, we discuss the concept of optical depth for a given catalogue or for a limiting magnitude.

The Manchester Microlensing Conference: The 12th International Conference and ANGLES Microlensing Workshop

January 21-25 2008

Manchester, $U K$

\footnotetext{
* Speaker.

${ }^{\dagger}$ On behalf of the EROS collaboration.
} 


\section{Introduction}

After the first discoveries of microlensing effects [1][2][3], the EROS team has performed extensive microlensing surveys from 1996 to 2003, that monitored the Magellanic clouds, and large regions in the galactic plane. Our team has devoted about $15 \%$ of the observing time during 7 seasons to search for microlensing events toward the Galactic Spiral Arms (GSA), as far as 55 degrees in longitude away from the Galactic center. In our previous publications [4][5] (hereafter refered as papers I and II) describing the detection of respectively 3 and 7 events, our attention was called on a possible optical depth asymmetry, emphasized by an asymetric event dynamics with respect to the Galactic center. This marginal effect (a 9\% probability to be accidental) could be interpreted as an indication of a long Galactic bar. Its investigation required a significant increase in the number of events.

In addition to the observing time increase (more than a factor 2), we improved our catalogue of monitored stars by increasing the limiting magnitude as well as by recovering some fields and subfields that were not analyzed previously. These improvements allowed us to recover another factor of two in sensitivity. Moreover the discrimination power for microlensing event identification has been significantly increased, partly due to the fact that the light curves are longer, and thus provide a better rejection of recurrent variable objects.

\section{Experimental setup and observations}

The telescope, the camera and the observations, as well as the operations and data reduction are described in paper I and references therein. The 29 fields that have been monitored in four different regions ( $\beta$ Sct, $\gamma$ Sct, $\gamma$ Nor and $\theta$ Mus) are shown in figure 1. Taking into account the dead zones, the lower efficiency sectors of our CCDs and the blind zones around the brightest stars, we estimate that $75 \pm 4 \%$ of the total CCD area $\left(0.95 \mathrm{deg}^{2}\right)$ was effectively sensitive. We took exposures of $120 \mathrm{~s}$ towards $\beta$ Sct (field $=4.3 \mathrm{deg} .{ }^{2}$ ), $\gamma$ Sct (field $=3.6 \mathrm{deg}^{2}{ }^{2}$ ) and $\gamma$ Nor (field $=8.4 \mathrm{deg}^{2}{ }^{2}$ ) and $180 \mathrm{~s}$ towards $\theta$ Mus (field $=3.8 \mathrm{deg} .{ }^{2}$ ). The observations span a period of $T_{o b s}=2325$ days, starting July 1996 and ending October 2002; 369 measurements per field were obtained on average in each of the $R_{E R O S}$ and $B_{E R O S}$ bands, which are related to the Cousins I and Johnson $\mathrm{V}$ magnitudes through the following colour equations, to a precision of $\sim 0.1 \mathrm{mag}$ :

$$
R_{E R O S}=I_{C}, \quad B_{E R O S}=V_{J}-0.4\left(V_{J}-I_{C}\right) .
$$

The catalogues of monitored stars have been produced following the procedure described in papers I and II. The objects in the catalogues considered below are identified and unambiguously associated in both colors. They have at least one good quality measurement in each color (fit of the Point Spread Function). We have removed objects that are close to a very bright star.

\section{The catalogues}

The seven season data set contains 12.9 million objects: 3.0 toward $\beta$ Sct, 2.4 toward $\gamma$ Sct, 5.2 toward $\gamma$ Nor and 2.3 toward $\theta$ Mus. The number of monitored stars was increased by $\sim 50 \%$ since the time of papers I and II, by producing a richer catalogue from a wider choice of good 


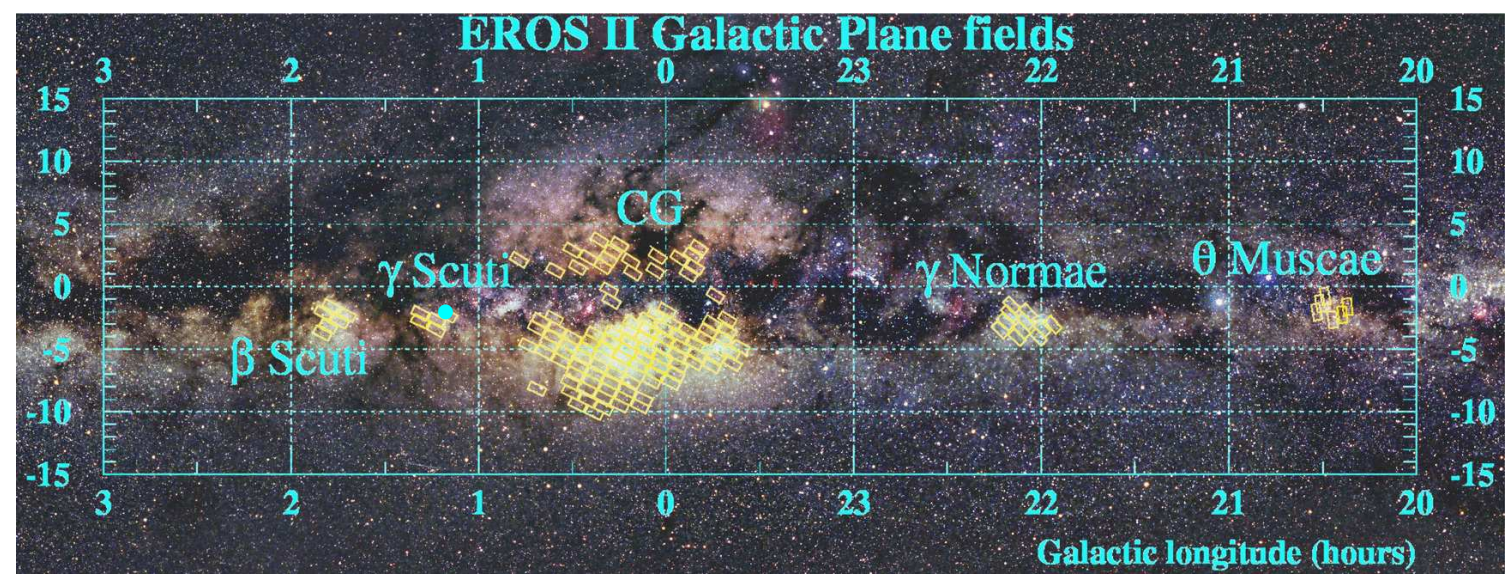

Figure 1: The Galactic plane fields (Galactic coordinates) monitored by EROS superimposed on the image of the Milky-way. The locations of our fields toward the spiral arms, as well as our Galactic bulge fields (not discussed in this paper) are shown. The large blue dot towards $\gamma$ Sct indicates the position of the HST field used to estimate our star detection efficiency (see text).

quality images than available before. We were also able to solve some technical problems that prevented us to produce the catalogue for some fields [6][7]. The recovered stars are mainly faint stars that do not permit a high microlensing sensitivity. The global pattern of the colour-magnitude diagrams follows the reddening versus absorption lines expected from a distance-distributed stellar populations.

From a comparison with HST images, we have estimated our star detection efficiency as a function of the star magnitude (see figure 2). This efficiency is the probability for a star to be detected as the main contributor to an EROS object in an active region of the CCD-array. It refers to the effective field (i.e. $0.71 \mathrm{deg}^{2}$ for the full mosaic). A star can also have a minor contribution to the flux of an EROS object, as a result of a blending effect. Such a blending limits the detection efficiency, even for bright stars, whenever such stars are blended with similar or brighter ones.

\section{The search for lensed stars}

The general technique to select microlensing events in our sample of light curves is the same as the one of papers I and II. We used the same non specific prefiltering described in paper II, and preselected the $\sim 15 \%$ most variable light curves. We searched for bumps and selected the light-curves displaying single significant bumps, simultaneously in the two colours. The candidate selection is then based on the fit quality $\left(\chi^{2}\right)$ and on variables obtained by using the $t_{0}$ (time of maximum magnification), $t_{E}$ (Einstein duration) and $u_{0}$ (impact parameter) fitted parameters. In particular, we select light curves with $\Delta \chi_{B}^{2}+\Delta \chi_{R}^{2}>60$, where $\Delta \chi^{2}$ is the $\chi^{2}$ improvement from a stable star fit to a microlensing fit. 27 microlensing candidates are selected by this selection process. Their $I_{C}$ magnitudes and colours $\left(V_{J}-I_{C}\right)$ are shown in figure 5 together with a set of points representing the population obtained after selection of simulated events. 


\section{Optical depth studies}

In the following, one should keep in mind that the impact on the optical depths of events with $t_{E}>700$ days cannot be estimated by EROS. To obtain reliable optical depth estimates, we use a sub-sample of good quality candidates, assumed to be free of the background that have been identified towards the other EROS targets. In this purpose, we only keep the candidates that have a fitted impact parameter $u_{0}<0.7$ (from the fit assuming a point-like source and a pointlike deflector with a constant speed). This is approximately equivalent to request the maximum magnification to be $A_{\max }>1.68$. As for our previous papers, we estimate our detection efficiency using the technique of the superposition of simulated events on experimental light curves from an unbiased sub-sample of our catalogue. Events are simulated as point-source, point-lens constant speed microlensing, with parameters uniformly spanning a domain largely exceeding the domain of experimental sensitivity ( $u_{0}$ up to 2, 1 day $<t_{E}<900$ days, $t_{0}$ generated from 150 days before the first observation to 150 days after the last) but efficiencies are normalized to the number of events generated up to $u_{0}=1$. Figure 3 shows the EROS efficiency as a function of $t_{E}$ averaged over all the other parameters, for each monitored direction. The optical depth estimates based on the 22 events
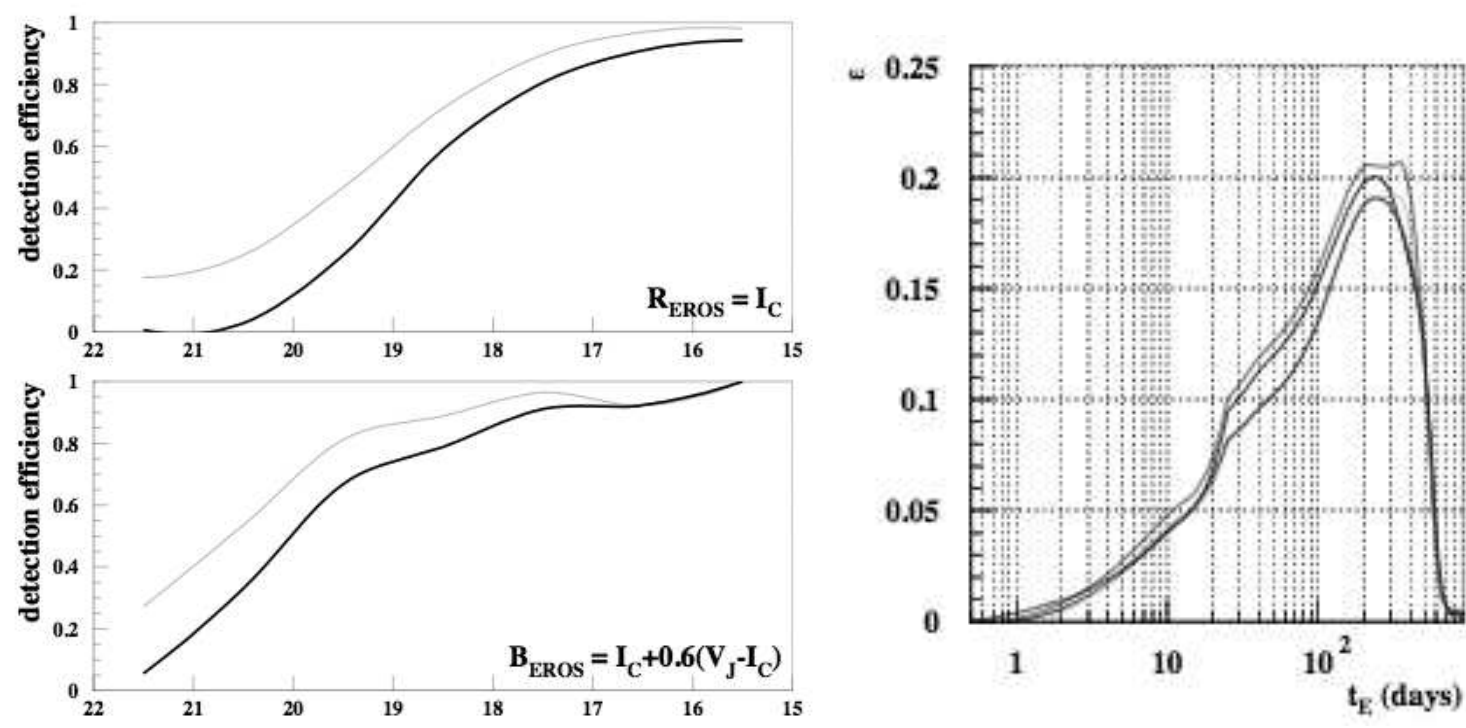

Figure 2: Left: star detection efficiencies versus $R_{E R O S}=I_{C}$ and versus $B_{E R O S}=I_{C}+0.6\left(V_{J}-I_{C}\right)$. The efficiency to detect a star in both EROS colours is the product of the two efficiencies. The thin line shows the probability for an HST star to contribute to an EROS object i.e. to be closer than 1 arcsec to such an object. The thick line gives the probability for an HST star to be the first contributor to the flux of an EROS object found within 1 arcsec.

Figure 3: Right: Microlensing detection efficiency as a function of $t_{E}$, averaged over all the other parameters.

with $u_{0}<0.7$ are given in the upper part of Table 1 . The average over all directions $\langle\tau\rangle_{\text {fields }}$ is defined as the proportion of stars covered by an Einstein disk. Fig. 7 shows the variation of $\langle\tau\rangle_{\text {fields }}$ with the threshold magnitude $I_{c}$ of the sources and the maximum color index $V_{J}-I_{C}$. An interesting use of these figures is the possibility to extract $\tau$ for specific stellar populations, in 


\begin{tabular}{|c|c|c|c|c|c|}
\hline & $\theta$ Mus & $\gamma$ Nor & $\gamma$ Sct & $\beta$ Sct & \\
\hline$\overline{b^{\circ}}$ & -1.46 & -2.42 & -2.09 & -2.15 & \\
\hline$\overline{l^{\circ}}$ & 306.56 & 331.09 & 18.51 & 26.60 & \\
\hline \multicolumn{5}{|c|}{ Observations } & All \\
\hline$\tau$ & $.68_{-.43}^{+.52}$ & $.48_{-.16}^{+.18}$ & $.69_{-.25}^{+.38}$ & $.28_{-.18}^{+.21}$ & $.50_{-.11}^{+.11}$ \\
\hline$N$ & 3 & 10 & 6 & 3 & 22 \\
\hline$\overline{t_{\mathrm{E}}}$ & 97.2 & 56.6 & 46.9 & 59.1 & $(59.1)$ \\
\hline$\sigma_{t_{\mathrm{E}}}$ & 80.3 & 27.9 & 13.7 & 8.3 & $(36.7)$ \\
\hline \multicolumn{5}{|c|}{$\tau$ from published models } & $\chi_{\text {model }}^{2}$ \\
\hline A & 0.32 & 0.48 & 0.79 & 0.60 & 2.9 \\
\hline +spiral & 0.56 & 0.69 & 1.07 & 0.83 & 9.3 \\
\hline B & 0.34 & 0.51 & 0.85 & 0.64 & 3.6 \\
\hline +spiral & 0.61 & 0.72 & 1.13 & 0.90 & 11.9 \\
\hline $\mathrm{C}$ & 0.47 & 0.78 & 1.11 & 0.95 & 14.3 \\
\hline +spiral & 0.71 & 1.18 & 1.43 & 1.23 & 39.4 \\
\hline \multicolumn{5}{|c|}{$\overline{t_{\mathrm{E}}}$ from published model } & \multirow{7}{*}{2.2} \\
\hline B & 45 & 28 & 25 & 27 & \\
\hline \multicolumn{5}{|c|}{ Predictions from model 1} & \\
\hline$\tau$ & 0.34 & 0.49 & 0.65 & 0.56 & \\
\hline$N$ & 1.1 & 3.6 & 1.6 & 1.4 & \\
\hline$\overline{t_{\mathrm{E}}}$ & 72 & 67 & 53 & 66 & \\
\hline$\sigma_{t_{\mathrm{E}}}$ & 48 & 45 & 42 & 47 & \\
\hline
\end{tabular}

Table 1: Observed and expected optical depth $\tau\left(\times 10^{6}\right)$, number of events $N$, average $\overline{t_{\mathrm{E}}}$, dispersion $\sigma_{t_{\mathrm{E}}}$ in days for each monitored direction, assuming that the distance to source stars is $7 \mathrm{kpc}$.

Quantitative comparisons of measured $\tau$ with the predictions of [8] (A), [9] (B), [10] (C) and including the effects of spiral structure [11], and with the simple Galactic model described in the text.

particular to consider only the brightest stars with $I_{C}<18.5$, or avoid the contamination of remote stars that are redder and more probably lensed. One advantage of considering only the brightest stars with $I_{C}<18.5$ is that these bright sources suffer less from blending. Moreover, our catalogue can be considered as almost complete at this magnitude, as our star detection efficiency is large (see Fig. 2) and the magnitude distribution peaks at $\sim 19$. Using such a sub-catalogue of bright stars should make the interpretation easier within a galactic model framework as we will discuss hereafter.

\subsection{First order comparisons with simple models}

We will only consider here a direct comparison with published optical depth calculations and with home-made estimates from a naive galactic model (without a thick disk). For the Galactic bulge, we take the parameters from [12] and [13] with $\Phi=45^{\circ}$ as the inclination of the bulge to the Galactic center line of sight, $a=1.97 k p c, b=0.3 k p c, c=0.25 k p c, M_{B}=2.4 \times 10^{10} M_{\odot}$. The thin disk has $\Sigma=50 M_{\odot} \mathrm{pc}^{-2}, H=0.325 \mathrm{kpc}, R=3.5 \mathrm{kpc}$ and $M_{\text {thin }}=4.3 \times 10^{10} M_{\odot}$. We completely neglect any contribution from the halo to the optical depth, in the light of the latest EROS results 

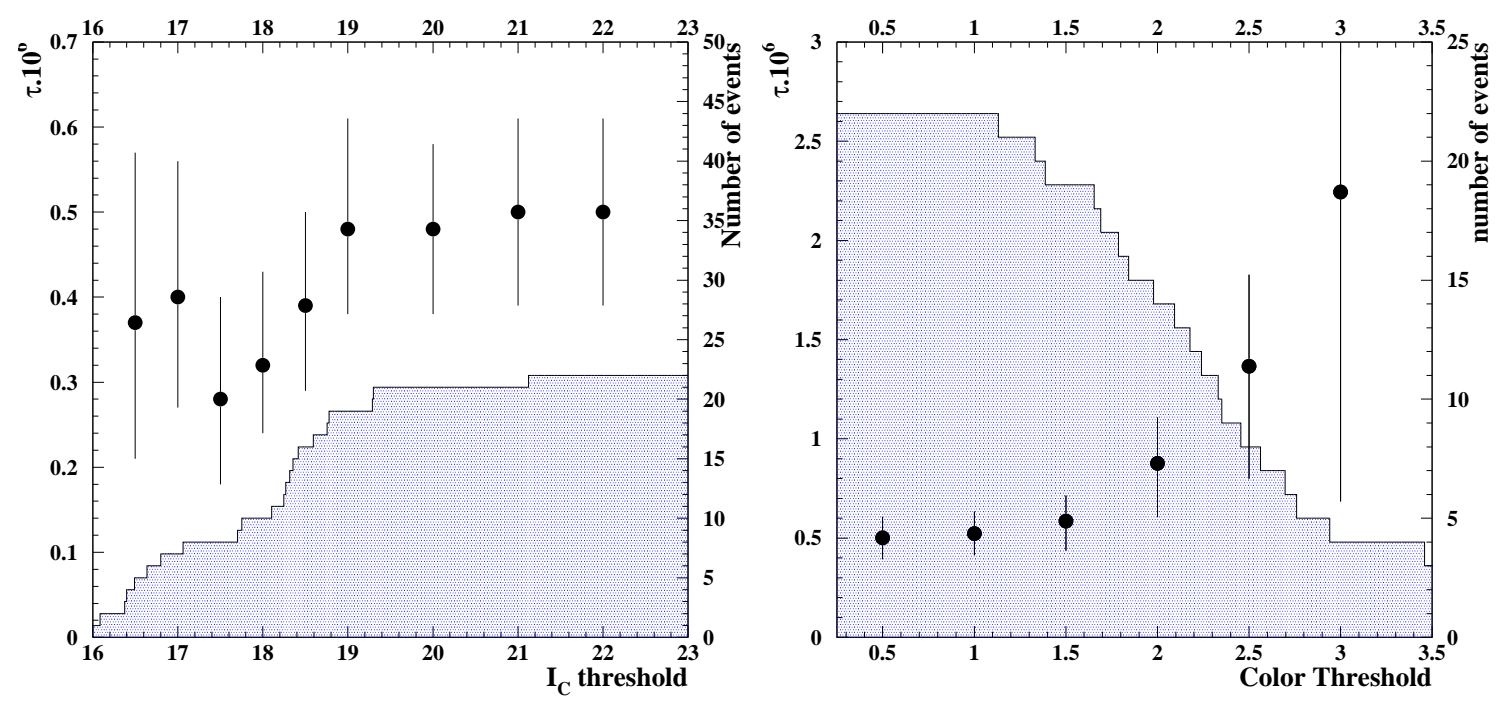

Figure 4: Number of selected candidates (right scale) and $\langle\tau\rangle_{\text {fields }}$ (left scale multiplied by $10^{6}$ ) for the sub-samples of stars brighter than the $I_{C}$ threshold (left) or redder than the color index $\left(V_{J}-I_{C}\right)$ threshold (right). Only statistical errors are plotted.

towards the Magellanic Clouds [14]. The different models can be compared through the value of:

$$
\chi_{\text {model }}^{2}=\sum_{\text {targets } i} \frac{\left(\tau_{i}(\text { model })-\tau_{i}(\text { observed })\right)^{2}}{\sigma_{i}^{2}}
$$

where $\sigma_{i}$ is taken as the largest error interval of $\tau_{i}$ determination. The simplest model is clearly favoured by the data and also model A without spiral structure. We cannot draw any firm conclusion from our simple model, as we know that it is not a realistic description, because all targets are supposed to be at the same distance. Nevertheless, it seems that "heavy" models trying to include thick disk or any spiral structure are in a difficult position.

\section{Guidelines for further interpretation}

As we always emphasized when presenting previous results towards the spiral arms, the poorly known distances of the monitored sources complicate the optical depth interpretation. Therefore, we provide here guidelines to properly interpret our optical depth estimates within a galactic model framework.

\subsection{The concept of catalogue optical depth}

The optical depth $\tau$ toward a source depends on its distance ; in contrast with LMC, SMC and the galactic center red giant clump, the monitored sources from the galactic spiral arms span a wide range of distances. Fig. 6 shows the expected optical depth as a function of the Galactic longitude $l$, for different target distances, using our simple model. One clearly sees that the near side of the bar increases the optical depth towards nearby stars, but on the contrary, the optical 

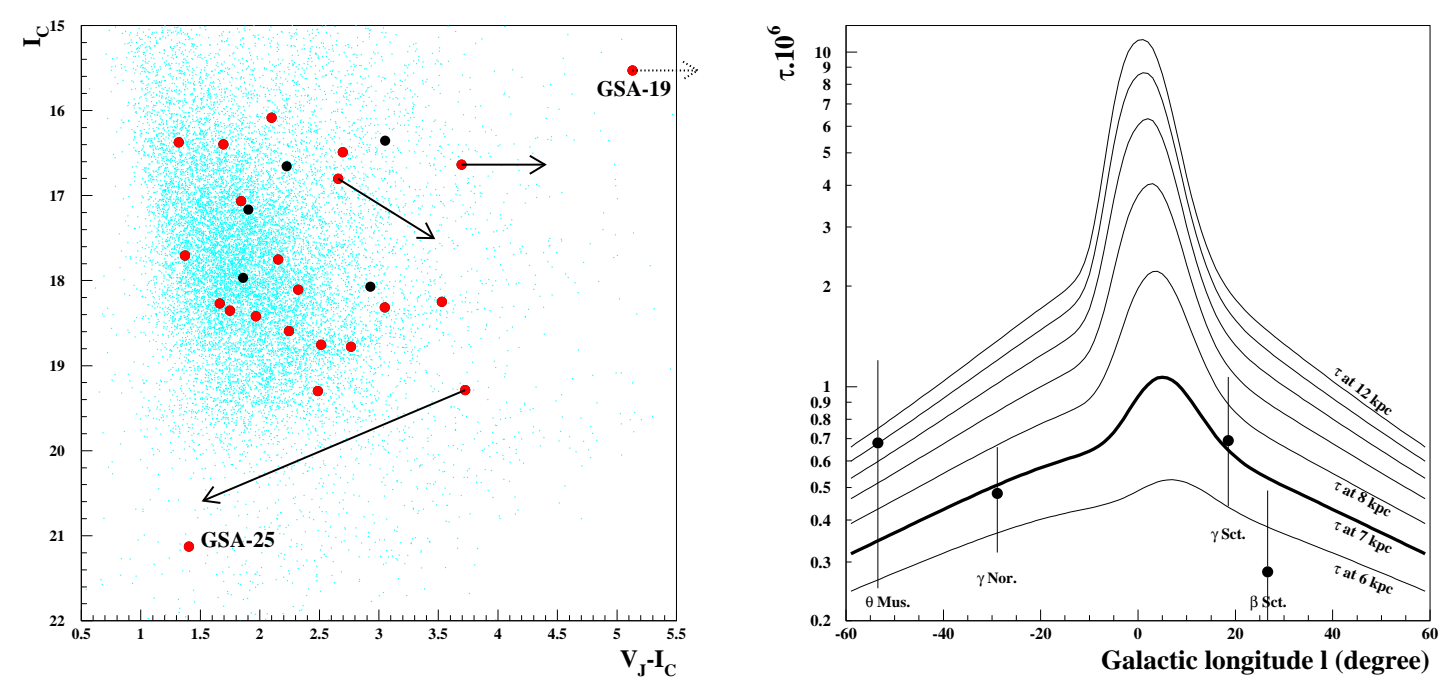

Figure 5: Left. Colour-magnitude diagram of the simulated events satisfying the analysis criteria (small dots) and the detected candidates (big dots). The arrows indicate the position of the magnified star in the case of blend. The red dots correspond to events that are used for the optical depth estimates.

Figure 6: Right. Expected optical depth for sources located at 6, 7, 8, 9, 10, 11 and $12 \mathrm{kpc}$ (from lowest to highest curve) at galactic latitude $b=-2.5^{\circ}$ as a function of the galactic longitude. The measured optical depths are quoted for our 4 targets.

depth towards remote stars is larger in the direction of the far side of the bar. EROS catalog includes contributions of stars located at every distance, and the measured optical depth is the average over the monitored sources distance distribution. Establishing the distance distribution of the sources through individual spectrophotometric measurements would require an enormous amount of complementary observations. Therefore we define the concept of "catalogue optical depth" $\tau_{c a t}$, that is relative to our catalogue of monitored stars, defined as the fraction of stars of the catalogue that undergo a magnification $A>1.34$. Such a measured optical depth can be compared with the depth derived from a lens and source distribution model as follows: first, one has to generate a synthetic source catalogue that matches the observed catalogue; then one can use the generated source distance distributions to estimate the average optical depth and compare with the measurements. This procedure will be described in more detail in [15].

\subsection{Estimating the average optical depth expected in the EROS catalogue from a model}

The pre-requisite for the optical depth study is that the statistical distribution of the physical observables (luminosity, colour and density per solid angle) for the synthesized source catalogue should fit the observed one. For this purpose, the colour-magnitude diagrams of our catalogues ([15] to be published) and the star detection efficiency of Fig. 2 2 should be used. After the synthesis of a satisfactory catalogue is performed, one can compute the average optical depth over the catalogued stars, from the distances provided by the model, and then compare with the measure- 
ments. With this procedure, the relative contribution of a fixed distance modelled population is automatically set through the use of the EROS star detection efficiency.

For easier comparisons, we provide here two "catalogue optical depths": the first one $\tau_{\text {complete }}=$ $0.50 \pm 0.10$ is relative to our entire catalogue, and the second one $\tau\left(I_{c}<18.5\right)=0.39_{-.10}^{+.10}$ is estimated on the sub-set of stars with apparent magnitude $I_{c}<18.5$, for which we believe our catalogue to be almost complete (see Fig.2).

\section{Conclusions}

The microlensing search of EROS2 towards the transparent windows of the spiral arms gives optical depths that are consistent with the simpliest galactic model. A more complete interpretation taking into account the distance dispersion of the monitored sources needs a model that synthetises the EROS catalogues. With such a modelisation, one will be able to make a proper use of the event duration distributions. The instrument that has currently the best capability to improve our knowledge of the microlensing towards the galactic plane is the wide-field infrared camera of VISTA. Observations in infrared will allow astronomers to monitor stars through the dust, making them free of the transparent windows that were limiting the EROS fields.

Acknowledgements: We are grateful to D. Lacroix and the technical staff at the Observatoire de Haute Provence and to A. Baranne for their help in refurbishing the MARLY telescope and remounting it in La Silla. We are also grateful for the support given to our project by the technical staff at ESO, La Silla. We thank J.F. Lecointe for assistance with the online computing. We wish to thank also C. Nitschelm for his contribution to the data taking.

\section{References}

[1] Aubourg É., Bareyre P., Bréhin S. et al. (EROS Coll.), 1993, Nat 365, 623.

[2] Alcock C., Akerlof C.W., Allsman R.A. et al. (MACHO Coll.), 1993, Nat 365, 621.

[3] Udalski A., Szymański M., Kaluzny J. et al. (OGLE Coll.), 1993, Act. Astr. 43, 289.

[4] Derue F., Afonso C., Alard C. et al. (EROS Coll.), 1999, A\&A 351, 87.

[5] Derue F., Afonso C., Alard C. et al. (EROS Coll.), 2001, A\&A 373, 126.

[6] Tisserand P., 2004, Ph.D. thesis, Université de Nice-Sophya Antipolis CEA DAPNIA-04-09-T.

[7] Rahal Y. R., 2003, Ph.D. thesis, Université Paris 6 LAL-CNRS/IN2P3 report 03-85.

[8] Binney J., Gerhard 0., \& Spergel D., 1997, MNRAS 288, 365.

[9] Dwek E., Arendt R.G., Hauser M.G. et al., 1995, ApJ 445, 716.

[10] Freudenreich H.T., 1998, ApJ 492, 495.

[11] Evans N.W. \& Belokurov V., 2002, ApJ 567, L119.

[12] Picaud S., Robin A.C., 2004, A\&A 428,891.

[13] Hamadache C., Le Guillou L., Tisserand P. et al., 2006, A\&A 454, 185.

[14] Tisserand P. et al.,2007, A\&A 469, 387.

[15] Rahal Y., et al., to be published. 